\title{
Sliding Mode Controller Design for Global CHAOS SYNCHRONIZATION OF COULLET SYSTEMS
}

\author{
Sundarapandian Vaidyanathan ${ }^{1}$ and Sivaperumal Sampath ${ }^{2}$ \\ ${ }^{1}$ Research and Development Centre, Vel Tech Dr. RR \& Dr. SR Technical University \\ Avadi, Chennai-600 062, Tamil Nadu, INDIA \\ sundarvtu@gmail.com \\ ${ }^{2}$ Institute of Technology, CMJ University Shillong, Meghalaya-793 003, INDIA \\ sivaperumals@gmail.com
}

\begin{abstract}
This paper derives new results for the design of sliding mode controller for the global chaos synchronization of identical Coullet systems (1981). The synchronizer results derived in this paper for the complete chaos synchronization of identical hyperchaotic systems are established using sliding control theory and Lyapunov stability theory. Since the Lyapunov exponents are not required for these calculations, the sliding mode control method is very effective and convenient to achieve global chaos synchronization of the identical Coullet systems. Numerical simulations are shown to illustrate and validate the synchronization schemes derived in this paper for the identical Coullet systems.
\end{abstract}

\section{KEYWORDS}

Sliding Mode Control, Chaos, Chaotic Systems, Chaos Synchronization, Coullet Systems.

\section{INTRODUCTION}

Chaotic systems are dynamical systems that are highly sensitive to initial conditions. The sensitive nature of chaotic systems is commonly called as the butterfly effect [1]. Chaotic behaviour can be observed in many natural systems, such as weather models [2].

Chaos is an interesting nonlinear phenomenon and has been extensively studied in the last three decades. Small differences in initial conditions (such as those due to rounding errors in numerical computation) yield widely diverging outcomes for chaotic systems. Chaos theory has been applied to a variety of fields such as physical systems [3], chemical systems [4], ecological systems [4], secure communications [6-8], etc.

In most of the chaos synchronization approaches, the master-slave or drive-response formalism is used. If a particular chaotic system is called the master or drive system and another chaotic system is called the slave or response system, then the idea of the synchronization is to use the output of the master system to control the slave system so that the output of the slave system tracks the output of the master system asymptotically.

Since the pioneering work by Pecora and Carroll ([9], 1990), chaos synchronization problem has been studied extensively and intensively in the literature [9-38]. In the last two decades, various

DOI : $10.5121 /$ ijist.2012.2207 
International Journal of Information Sciences and Techniques (IJIST) Vol.2, No.2, March 2012

schemes have been successfully applied for chaos synchronization such as PC method [9], OGY method [10], active control method [11-18], adaptive control method [19-27], time-delay feedback method [28-29], backstepping design method [30-32], sampled-data feedback method [33], etc.

In this paper, we derive new results based on the sliding mode control [34-38] for the global chaos synchronization of identical Coullet systems ([39], 1981). In robust control systems, the sliding mode control method is often adopted due to its inherent advantages of easy realization, fast response and good transient performance as well as its insensitivity to parameter uncertainties and external disturbances.

This paper has been organized as follows. In Section 2, we describe the problem statement and our methodology using sliding mode control (SMC). In Section 3, we discuss the global chaos synchronization of identical Coullet systems. In Section 4, we summarize the main results obtained in this paper.

\section{Problem Statement and OUR Methodology using SMC}

In this section, we describe the problem statement for the global chaos synchronization for identical chaotic systems and our methodology using sliding mode control (SMC).

Consider the chaotic system described by

$$
\dot{x}=A x+f(x)
$$

where $x \in R^{n}$ is the state of the system, $A$ is the $n \times n$ matrix of the system parameters and $f: R^{n} \rightarrow R^{n}$ is the nonlinear part of the system.

We consider the system (1) as the master or drive system.

As the slave or response system, we consider the following chaotic system described by the dynamics

$$
\dot{y}=A y+f(y)+u
$$

where $y \in R^{n}$ is the state of the system and $u \in R^{m}$ is the controller to be designed.

If we define the synchronization error as

$$
e=y-x
$$

then the error dynamics is obtained as

$$
\dot{e}=A e+\eta(x, y)+u,
$$

where

$$
\eta(x, y)=f(y)-f(x)
$$

The objective of the global chaos synchronization problem is to find a controller $u$ such that 


$$
\lim _{t \rightarrow \infty}\|e(t)\|=0 \quad \text { for all } e(0) \in R^{n} .
$$

To solve this problem, we first define the control $u$ as

$$
u=-\eta(x, y)+B v
$$

where $B$ is a constant gain vector selected such that $(A, B)$ is controllable.

Substituting (6) into (4), the error dynamics simplifies to

$$
\dot{e}=A e+B v
$$

which is a linear time-invariant control system with single input $v$.

Thus, the original global chaos synchronization problem can be replaced by an equivalent problem of stabilizing the zero solution $e=0$ of the system (7) by a suitable choice of the sliding mode control. In the sliding mode control, we define the variable

$$
s(e)=C e=c_{1} e_{1}+c_{2} e_{2}+\cdots+c_{n} e_{n}
$$

where $C=\left[\begin{array}{llll}c_{1} & c_{2} & \cdots & c_{n}\end{array}\right]$ is a constant row vector to be determined.

In the sliding mode control, we constrain the motion of the system (7) to the sliding manifold defined by

$$
S=\left\{x \in R^{n} \mid s(e)=0\right\}
$$

which is required to be invariant under the flow of the error dynamics (7).

When in sliding manifold $S$, the system (7) satisfies the following conditions:

$$
s(e)=0
$$

which is the defining equation for the manifold $S$ and

$$
\dot{s}(e)=0
$$

which is the necessary condition for the state trajectory $e(t)$ of (7) to stay on the sliding manifold $S$.

Using (7) and (8), the equation (10) can be rewritten as

$$
\dot{s}(e)=C[A e+B v]=0
$$

Solving (11) for $v$, we obtain the equivalent control law

$$
v_{\mathrm{eq}}(t)=-(C B)^{-1} C A e(t)
$$


where $C$ is chosen such that $C B \neq 0$.

Substituting (12) into the error dynamics (7), we obtain the closed-loop dynamics as

$$
\dot{e}=\left[I-B(C B)^{-1} C\right] A e
$$

The row vector $C$ is selected such that the system matrix of the controlled dynamics $\left[I-B(C B)^{-1} C\right] A$ is Hurwitz, i.e. it has all eigenvalues with negative real parts. Then the controlled system (13) is globally asymptotically stable.

To design the sliding mode controller for (7), we apply the constant plus proportional rate reaching law

$$
\dot{s}=-q \operatorname{sgn}(s)-k s
$$

where $\operatorname{sgn}(\cdot)$ denotes the sign function and the gains $q>0, k>0$ are determined such that the sliding condition is satisfied and sliding motion will occur.

From equations (11) and (14), we can obtain the control $v(t)$ as

$$
v(t)=-(C B)^{-1}[C(k I+A) e+q \operatorname{sgn}(s)]
$$

which yields

$$
v(t)= \begin{cases}-(C B)^{-1}[C(k I+A) e+q], & \text { if } s(e)>0 \\ -(C B)^{-1}[C(k I+A) e-q], & \text { if } s(e)<0\end{cases}
$$

Theorem 1. The master system (1) and the slave system (2) are globally and asymptotically synchronized for all initial conditions $x(0), y(0) \in R^{n}$ by the feedback control law

$$
u(t)=-\eta(x, y)+B v(t)
$$

where $v(t)$ is defined by $(15)$ and $B$ is a column vector such that $(A, B)$ is controllable. Also, the sliding mode gains $k$, q are positive.

Proof. First, we note that substituting (17) and (15) into the error dynamics (4), we obtain the closed-loop error dynamics as

$$
\dot{e}=A e-B(C B)^{-1}[C(k I+A) e+q \operatorname{sgn}(s)]
$$

To prove that the error dynamics (18) is globally asymptotically stable, we consider the candidate Lyapunov function defined by the equation

$$
V(e)=\frac{1}{2} s^{2}(e)
$$

Differentiating $V$ along the trajectories of (18) or the equivalent dynamics (14), we get 


$$
\dot{V}(e)=s(e) \dot{s}(e)=-k s^{2}-q \operatorname{sgn}(s) s
$$

We note that $\dot{V}$ is a negative definite function on $R^{n}$.

This calculation shows that $V$ is a globally defined, positive definite, Lyapunov function for the error dynamics (18), which has a globally defined, negative definite time derivative $\dot{V}$.

Thus, by Lyapunov stability theory [40], it is immediate that the error dynamics (18) is globally asymptotically stable for all initial conditions $e(0) \in R^{n}$.

Hence, it follows that the master system (1) and the slave system (2) are globally and asymptotically synchronized for all initial conditions $x(0), y(0) \in R^{n}$.

This completes the proof.

\section{Sliding Controller Design for Global ChaOs Synchronization OF IDENTICAL COULLET SySTEMS}

\subsection{Theoretical Results}

In this section, we apply the sliding mode control results of Section 2 to derive state feedback control laws for the global chaos synchronization of identical Coullet systems ([39], 1981). The Coullet chaotic system is one of the paradigms of 3-D chaotic systems.

Thus, the master system is described by the Coullet dynamics

$$
\begin{aligned}
& \dot{x}_{1}=x_{2} \\
& \dot{x}_{2}=x_{3} \\
& \dot{x}_{3}=a x_{1}-b x_{2}-c x_{3}-x_{1}^{3}
\end{aligned}
$$

where $x_{1}, x_{2}, x_{3}$ are state variables and $a, b, c$ are positive, constant parameters of the system. The slave system is also described by the controlled Coullet dynamics

$$
\begin{aligned}
& \dot{y}_{1}=y_{2}+u_{1} \\
& \dot{y}_{2}=y_{3}+u_{2} \\
& \dot{y}_{3}=a y_{1}-b y_{2}-c y_{3}-y_{1}^{3}+u_{3}
\end{aligned}
$$

where $y_{1}, y_{2}, y_{3}$ are state variables and $u_{1}, u_{2}, u_{3}$ are the controllers to be designed.

The 3-D systems (21) and (22) are chaotic when

$$
a=5.5, b=3.5, c=1.0
$$

Figure 1 illustrates the strange attractor of the Coullet chaotic system. 


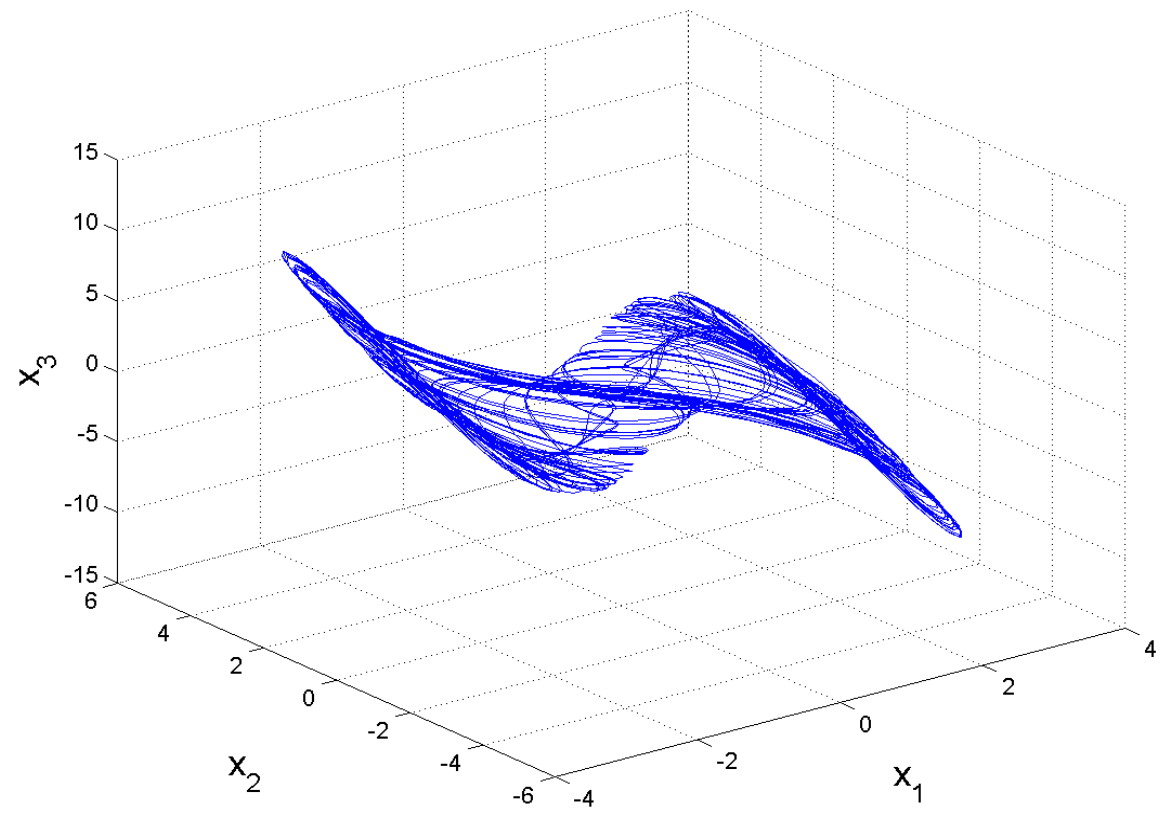

Figure 1. Strange Attractor of the Coullet System

The chaos synchronization error is defined by

$$
\begin{aligned}
& e_{1}=y_{1}-x_{1} \\
& e_{2}=y_{2}-x_{2} \\
& e_{3}=y_{3}-x_{3}
\end{aligned}
$$

The error dynamics is easily obtained as

$$
\begin{aligned}
& \dot{e}_{1}=e_{2}+u_{1} \\
& \dot{e}_{2}=e_{3}+u_{2} \\
& \dot{e}_{3}=a e_{1}-b e_{2}-c e_{3}-y_{1}^{3}+x_{1}^{3}+u_{3}
\end{aligned}
$$

We write the error dynamics (24) in the matrix notation as

$$
\dot{e}=A e+\eta(x, y)+u
$$

where

$$
A=\left[\begin{array}{ccc}
0 & 1 & 0 \\
0 & 0 & 1 \\
a & -b & -c
\end{array}\right], \quad \eta(x, y)=\left[\begin{array}{c}
0 \\
0 \\
-y_{1}^{3}+x_{1}^{3}
\end{array}\right] \text { and } u=\left[\begin{array}{l}
u_{1} \\
u_{2} \\
u_{3}
\end{array}\right]
$$

The sliding mode controller design is carried out as detailed in Section 2. 
First, we set $u$ as

$$
u=-\eta(x, y)+B v
$$

where $B$ is chosen such that $(A, B)$ is controllable.

We take $B$ as

$$
B=\left[\begin{array}{l}
1 \\
1 \\
1
\end{array}\right]
$$

In the chaotic case, the parameter values are

$$
a=5.5, b=3.5, c=1.0
$$

The sliding mode variable is selected as

$$
s=C e=\left[\begin{array}{lll}
10 & 4 & 1
\end{array}\right] e=10 e_{1}+4 e_{2}+e_{3}
$$

which makes the sliding mode state equation asymptotically stable.

We choose the sliding mode gains as

$$
k=5 \text { and } q=0.2
$$

We note that a large value of $k$ can cause chattering and an appropriate value of $q$ is chosen to speed up the time taken to reach the sliding manifold as well as to reduce the system chattering.

From Eq. (15), we can obtain $v(t)$ as

$$
v(t)=-3.7 e_{1}-1.7667 e_{2}-0.5333 e_{3}-0.0133 \operatorname{sgn}(s)
$$

Thus, the required sliding mode controller is obtained as

$$
u=-\eta(x, y)+B v
$$

where $\eta(x, y), B$ and $v(t)$ are defined as in the equations (26), (28) and (30).

By Theorem 1, we obtain the following result.

Theorem 2. The identical Coullet systems (21) and (22) are globally and asymptotically synchronized for all initial conditions with the sliding mode controller u defined by (31).

\subsection{Numerical Results}

For the numerical simulations, the fourth-order Runge-Kutta method with time-step $h=10^{-8}$ is used to solve the Coullet systems (21) and (22) with the sliding mode controller $u$ given by (31) 
International Journal of Information Sciences and Techniques (IJIST) Vol.2, No.2, March 2012

using MATLAB.

In the chaotic case, the parameter values are given by

$$
a=5.5, b=3.5, c=1.0
$$

The sliding mode gains are chosen as

$$
k=5 \text { and } q=0.2
$$

The initial values of the master system (21) are taken as

$$
x_{1}(0)=1, \quad x_{2}(0)=-4, \quad x_{3}(0)=2
$$

The initial values of the slave system (22) are taken as

$$
y_{1}(0)=-3, \quad y_{2}(0)=7, \quad y_{3}(0)=-8
$$

Figure 2 shows the complete synchronization of the identical Coullet systems (21) and (22).

Figure 3 shows the time-history of the synchronization error states $e_{1}(t), e_{2}(t), e_{3}(t)$.
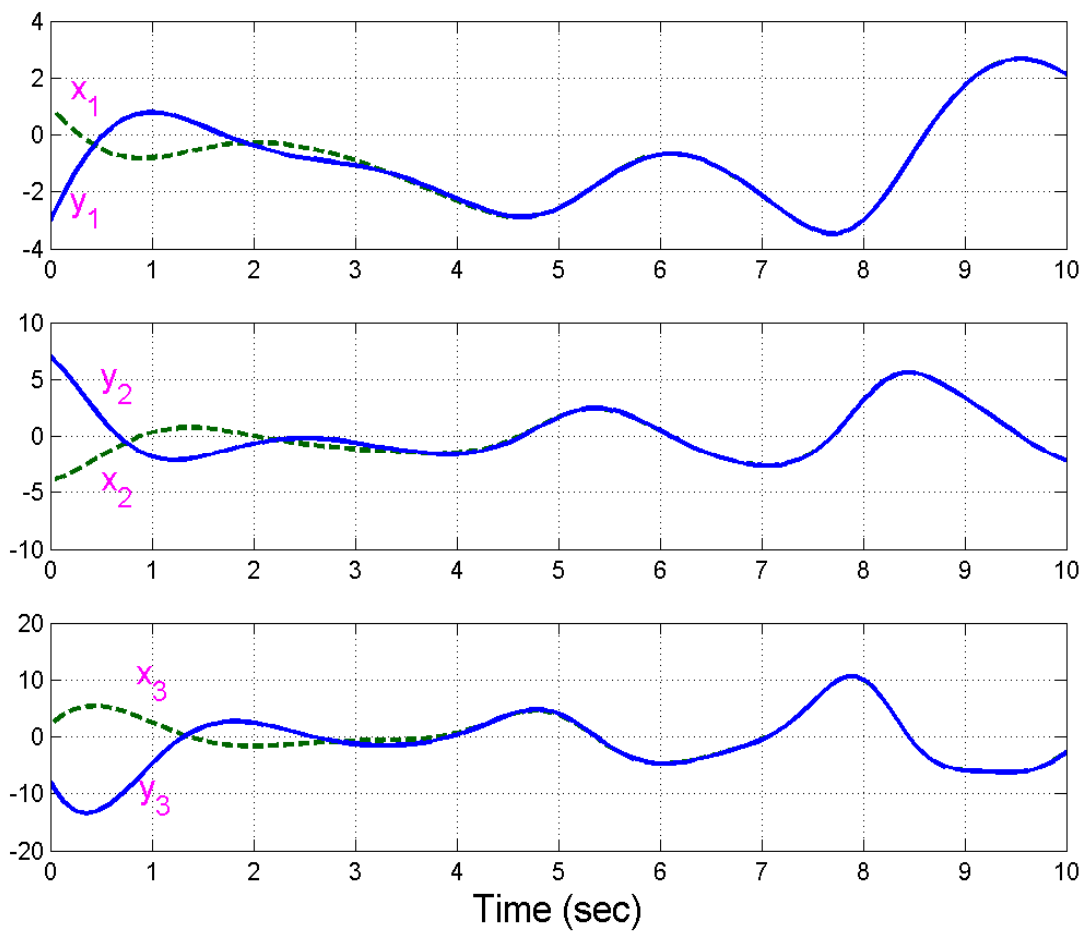

Figure 2. Complete Synchronization of Identical Coullet Systems 


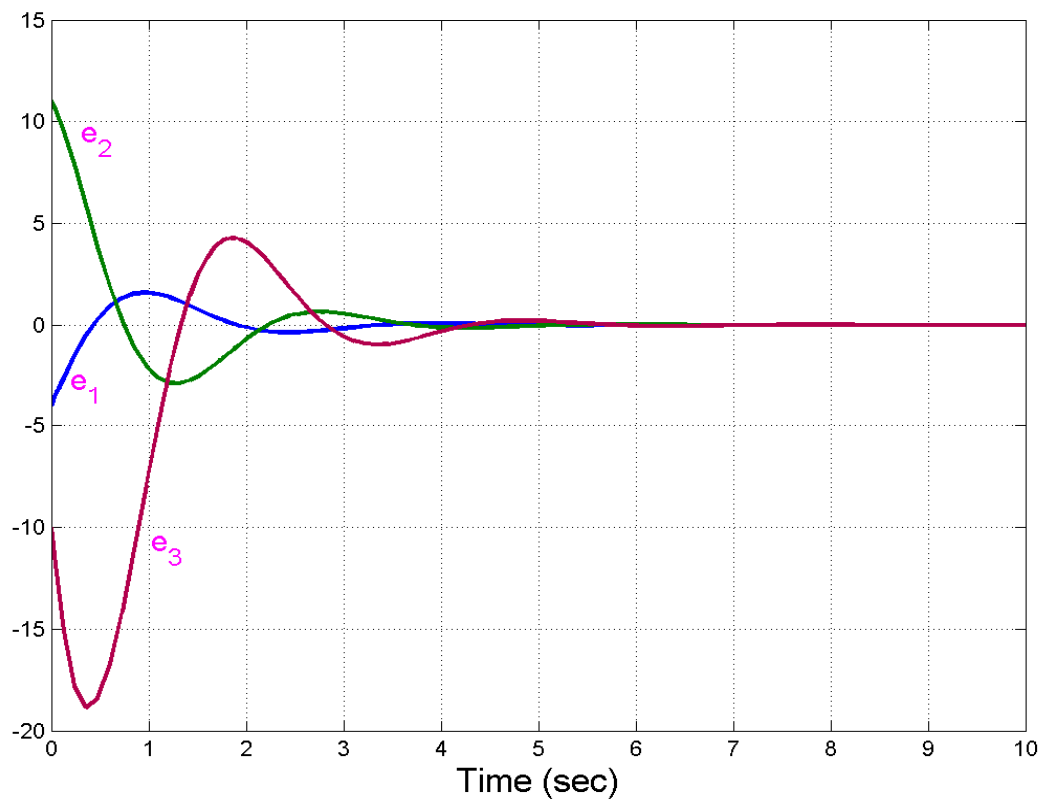

Figure 3. Time History of the Synchronization Error States $e_{1}, e_{2}, e_{3}$

\section{Conclusions}

In this paper, we have derived new results using Lyapunov stability theory for the global chaos synchronization using sliding mode control. As application of our sliding mode controller design, we derived new synchronization schemes for the identical Coullet systems (1981). Since the Lyapunov exponents are not required for these calculations, the sliding mode control method is very effective and convenient to achieve global chaos synchronization for the identical Coullet systems. Numerical simulations are also shown to illustrate the effectiveness of the synchronization results derived in this paper via sliding mode control.

\section{REFERENCES}

[1] Alligood, K.T., Sauer, T. \& Yorke, J.A. (1997) Chaos: An Introduction to Dynamical Systems, Springer, New York.

[2] Lorenz, E.N. (1963) “Deterministic nonperiodic flow,” J. Atmos. Phys. Vol. 20, pp 131-141.

[3] Lakshmanan, M. \& Murali, K. (1996) Nonlinear Oscillators: Controlling and Synchronization, World Scientific, Singapore.

[4] Han, S.K., Kerrer, C. \& Kuramoto, Y. (1995) "Dephasing and burstling in coupled neural oscillators," Physical Review Letters, Vol. 75, pp 3190-3193.

[5] Blasius, B., Huppert, A. \& Stone, L. (1999) "Complex dynamics and phase synchronization in spatially extended ecological system”, Nature, Vol. 399, pp 354-359.

[6] Cuomo, K.M. \& Oppenheim, A.V. (1993) "Circuit implementation of synchronized chaos with applications to communications," Physical Review Letters, Vol. 71, pp 65-68.

[7] Kocarev, L. \& Parlitz, U. (1995) "General approach for chaotic synchronization with applications to communication," Physical Review Letters, Vol. 74, pp 5028-5030. 
International Journal of Information Sciences and Techniques (IJIST) Vol.2, No.2, March 2012

[8] Tao, Y. (1999) "Chaotic secure communication systems - history and new results," Telecommun. Review, Vol. 9, pp 597-634.

[9] Pecora, L.M. \& Carroll, T.L. (1990) "Synchronization in chaotic systems", Physical Review Letters, Vol. 64, pp 821-824.

[10] Ott, E., Grebogi, C. \& Yorke, J.A. (1990) "Controlling chaos”, Physical Review Letters, Vol. 64, pp 1196-1199.

[11] Ho, M.C. \& Hung, Y.C. (2002) "Synchronization of two different chaotic systems using generalized active network," Physics Letters A, Vol. 301, pp 424-428.

[12] Huang, L., Feng, R. \& Wang, M. (2005) "Synchronization of chaotic systems via nonlinear control," Physical Letters A, Vol. 320, pp 271-275.

[13] Chen, H.K. (2005) "Global chaos synchronization of new chaotic systems via nonlinear control," Chaos, Solitons \& Fractals, Vol. 23, pp 1245-1251.

[14] Sundarapandian, V. \& Suresh, R. (2011) “Global chaos synchronization of hyperchaotic Qi and Jia systems by nonlinear control," International Journal of Distributed and Parallel Systems, Vol. 2, No. 2, pp 83-94.

[15] Sundarapandian, V. \& Karthikeyan, R. (2011) "Active controller design for global chaos antisynchronization of $\mathrm{Li}$ and Tigan chaotic systems," International Journal of Information Technology and Computer Science, Vol. 3, No. 4, pp 255-268.

[16] Sundarapandian, V. (2011) "Global chaos synchronization of Li and Lü chaotic systems by active nonlinear control," International Journal of Computer Information Systems, Vol. 2, No. 3, pp 2428 .

[17] Sundarapandian, V. (2011) “Anti-synchronization of Liu and Wang chaotic systems by active nonlinear control," International Journal of Computer Information Systems, Vol. 2, No. 6, pp 3237.

[18] Sundarapandian, V. (2011) "Anti-synchronization of Arneodo and Coullet systems by active nonlinear control," International Journal of Control Theory and Applications, Vol. 4, No. 1, pp 25-36.

[19] Liao, T.L. \& Lin, S.H. (1999) “Adaptive control and synchronization of Lorenz systems,” Journal of Franklin Institute, Vol. 326, pp 925-933.

[20] Chen, S.H. \& Lü, J. (2002) "Synchronization of an uncertain unified system via adaptive control," Chaos, Solitons \& Fractals, Vol. 14, pp 643-647.

[21] Yassen, M.T. (2003) "Adaptive control and synchronization of a modified Chua's circuit system," Applied Mathematics and Computation, Vol. 135, pp 113-120.

[22] Lu, J., Wu, X., Han, X. \& Lü, J. (2004) “Adaptive feedback synchronization of a unified chaotic system,” Physics Letters A, Vol. 329, pp 327-333.

[23] Hu, J., Chen, S. \& Chen, L. (2005) “Adaptive control for anti-synchronization of Chua's chaotic system,” Physics Letters A, Vol. 339, pp 455-460.

[24] Chen, X. \& Lu, J. (2007) “Adaptive synchronization of different chaotic systems with fully unknown parameters," Physics Letters A, Vol. 364, pp 123-128.

[25] Sundarapandian, V. (2011) "Adaptive control and synchronization of hyperchaotic Liu system," International Journal of Computer Science, Engineering and Information Technology, Vol. 1, No. 2, pp 29-40.

[26] Sundarapandian, V. (2011) "Adaptive control and synchronization of hyperchaotic NewtonLeipnik system," International Journal of Advanced Information Technology, Vol. 1, No. 3, pp 22-33.

[27] Sundarapandian, V. (2011) "Adaptive synchronization of hyperchaotic Lorenz and hyperchaotic Lü systems," International Journal of Instrumentation and Control Systems, Vol. 1, No. 1, pp 1-18. 
International Journal of Information Sciences and Techniques (IJIST) Vol.2, No.2, March 2012

[28] Park, J.H. \& Kwon, O.M. (2003) "A novel criterion for delayed feedback control of time-delay chaotic systems," Chaos, Solitons \& Fractals, Vol. 17, pp 709-716.

[29] Sun, M., Tian, L. \& Xu, J. (2006) "Time-delayed feedback control of the energy resource chaotic system,” International Journal of Nonlinear Science, Vol. 1, No. 3, pp 172-177.

[30] Wu, X. \& Lü, J. (2003) "Parameter identification and backstepping control of uncertain Lü system," Chaos, Solitons \& Fractals, Vol. 18, pp 721-729.

[31] Yu, Y. \& Zhang, S. (2002) “Adaptive backstepping control of the uncertain Lü system," Chinese Physics, Vol. 11, pp 1249-1253.

[32] Wu, Y., Zhou, X., Chen, J. \& Hui, B. (2009) "Chaos synchronization of a new 3D chaotic system," Chaos, Solitons \& Fractals, Vol. 42, pp 1812-1819.

[33] Zhao, J. \& J. Lu (2006) "Using sampled-data feedback control and linear feedback synchronization in a new hyperchaotic system," Chaos, Solitons \& Fractals, Vol. 35, pp 376-382.

[34] Slotine, J.E. \& Sastry, S.S. (1983) “Tracking control of nonlinear systems using sliding surface with application to robotic manipulators," International Journal of Control, Vol. 38, pp 465-492.

[35] Young, K.D., Utkin, V.I. \& Ozguner, Umit (1999) “A control engineer's guide to sliding mode control," IEEE Transactions on Control Systems Technology, Vol. 7, No. 3, pp 328-342.

[36] Sundarapandian, V. \& Sivaperumal, S. (2011) "Global chaos synchronization of hyperchaotic Chen systems by sliding mode control," International Journal of Engineering Science and Technology, Vol. 3, No. 5, pp 4265-4271.

[37] Sundarapandian, V. \& Sivaperumal, S. (2011) “Anti-synchronization of hyperchaotic Qi systems by sliding mode control," International Journal of Engineering Science and Technology, Vol. 3, No. 7, pp 5733-5739.

[38] Sundarapandian, V. \& Sivaperumal, S. (2011) “Anti-synchronization of Arneodo chaotic systems by sliding mode control," International Journal of Engineering Science and Technology, Vol. 3, No. 7, pp 5740-5747.

[39] Arneodo, A., Coullet, P. \& Tresser, C. (1981) "Possible new strange attractors with spiral structure," Commun. Math. Physics, Vol. 79, pp 573-579.

[40] Hahn, W. (1967) The Stability of Motion, Springer, New York.

\section{Authors}

Dr. V. Sundarapandian obtained his Doctor of Science degree in Electrical and Systems Engineering from Washington University, Saint Louis, USA under the guidance of Late Dr. Christopher I. Byrnes (Dean, School of Engineering and Applied Science) in 1996. He is currently Professor in the Research and Development Centre at Vel Tech Dr. RR \& Dr. SR Technical University, Chennai, Tamil Nadu, India. He has published over 240 refereed international publications. He has published over 100 papers in National Conferences and over 60 papers in International Conferences. He is the Editor-in-Chief of International Journal of Mathematics and Scientific Computing, International Journal of Instrumentation and Control Systems, International Journal

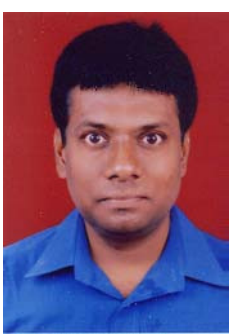
of Control Systems and Computer Modelling, International Journal of Information Technology, Control and Automation, etc. His research interests are Linear and Nonlinear Control Systems, Chaos Theory and Control, Soft Computing, Optimal Control, Process Control, Operations Research, Mathematical Modelling and Scientific Computing using MATLAB. He has delivered several Key Note Lectures on Linear and Nonlinear Control Systems, Chaos Theory and Control, Scientific Computing using MATLAB, SCILAB, etc. 
International Journal of Information Sciences and Techniques (IJIST) Vol.2, No.2, March 2012

Mr. S. Sivaperumal obtained his M.E. degree in VLSI Design and B.E. degree in Electronics and Communications Engineering from Anna University, Chennai in the years 2007 and 2005 respectively. He is currently Assistant Professor in the ECE Department, Vel Tech Dr. RR \& Dr. SR Technical University, Chennai, India. He is pursuing Ph.D. degree in Electronics and Communications Engineering from Institute of Technology, CMJ University, Shillong, Meghalaya, India. He has published many papers in refereed International Journals. He has also published papers on VLSI and Control Systems in National and International Conferences. His current research interests are Robotics, Communications, Control Systems and VLSI Design.

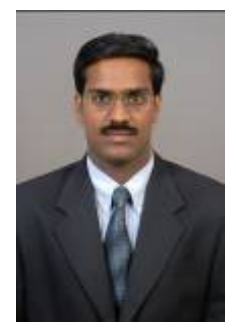

